\title{
Salivary Gland Oncocytoma
}

National Cancer Institute

\section{Source}

National Cancer Institute. Salivary Gland Oncocytoma. NCI Thesaurus. Code C5932.

A rare benign epithelial neoplasm composed of oncocytes, which are large cells with a large amount of granular eosinophilic cytoplasm containing numerous tightly-packed mitochondria. Approximately $85 \%$ to $90 \%$ occur in the parotid gland; most of the remainder occur in the submandibular gland. The peak incidence is in the seventh through ninth decades of life. In almost all cases, swelling is the only complaint of the patient. -2003 